Widefield Science and Technology for the SKA

SKADS CONFERENCE 2009

S.A. Torchinsky, A. van Ardenne, T. van den Brink-Havinga, A.J.J. van Es, A.J. Faulkner (eds.)

4-6 November 2009, Château de Limelette, Belgium

\title{
EMBRACE System Design and Realisation
}

\author{
G.W. Kant, E. van der Wal, M. Ruiter, and P. Benthem
}

ASTRON, Oude Hoogeveensedijk 4, NL-7991 PD Dwingeloo, The Netherlands *

\begin{abstract}
EMBRACE is an operational demonstrator for next generation radio telescopes. It demonstrates independent, multiple, wide field, wide band receiving antenna beams based on phased array technology. EMBRACE operates in the 500-1500 MHz frequency range and consist of two aperture array stations. Currently, the Westerbork and the Nançay stations are being realised. The design and realisation has been performed in a multidisciplinary project with strong emphasis on the front end. During the project multiple design cycles were performed to find an optimal prototype suitable for large volume production. A major building block of the array is denoted as a tile. The final configuration of a tile has one control board with six identical hex-modules supporting 144 Vivaldi elements. Apart from the electronics, mechanics play a major role. One example is a radome concept which has been developed to combine a temperature stabilised environment for the front-end and maintaining an electromagnetic transparent view on the sky. In this paper a system overview of EMBRACE is presented and the background of the main design choices is discussed. Both engineering and astronomical testing can be performed concurrently. This is possible as each logical tile forms an autonomous functional item providing an operational signal path. Furthermore, the digital back-end can be partitioned. All required components have been designed, tested and verified using a bottom up approach. This approach resulted in a gradually growing complexity of the system while adding more verified components. The production of the subsystems included technologies like low cost laser-cut antenna assemblies, extruded profiles, printed circuit boards, tile frames and the radome itself. All hardware manufacturing has finished. Currently more than half of the Westerbork array has been built and the realisation of the Nançay station is ramping up.
\end{abstract}

\section{Introduction}

For classic radio astronomy telescopes, metallic parabolic surfaces are used to collect the power of incident electromagnetic field in horn antennas. The incident electromagnetic field induces currents on the surface of the parabola and the induced currents create, on their turn, a scattered field which has a focus point at the horn. Since the phase relation of the induced currents on the surface is determined by the parabolic shape, pointing of the beam has to be done in a mechanical fashion. Large parabolic reflectors require mechanical mount structures with motors to steer the whole structure. Also, the accuracy of the parabola requires a complex backing structure behind the parabolic surface withstanding gravity and wind load. As a whole the result is that for frequencies below $2 \mathrm{GHz}$ a phased array approach becomes a competitive alternative for the classic parabolic radio telescope.

With an aperture array like the one shown in Fig. 1, the incident field induces currents on the antenna elements of the phased array. By altering the phases of the induced currents and adding the resulting signals, the operation of a parabolic antenna is synthesised. Because at element level the currents can be altered, the resulting beam can be pointed into any direction on the sky. This way the mechanical pointing of a parabolic antenna is replaced by silicon devices in the aperture array case.

It is not only possible to alter the phases of the signals, also the amplitudes can be controlled at element level. Any amplitude taper can be selected over the array enabling tight control of the side lobe levels of the resulting array pattern. High

* This work was supported by the European Commission Framework Program 6, Project SKADS, Square Kilometer Array Design Studies (SKADS), contract no. 011938.

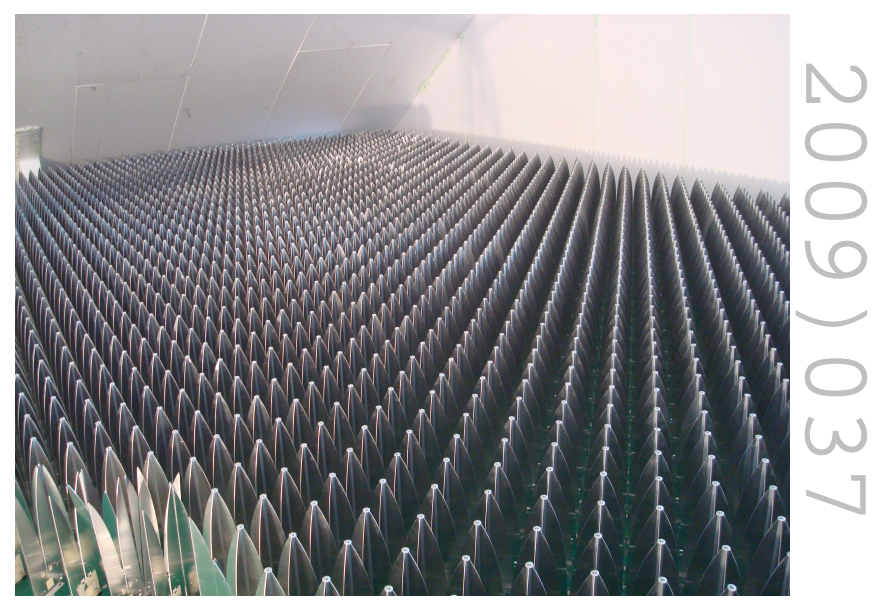

Fig. 1: A picture giving an impression of the phased array inside the radome at the Westerbork EMBRACE station. Shown are the aluminium Vivaldi radiators in a dual polarisation configuration of about a quarter of the array.

aperture efficiency can be traded for low side lobe levels in an electronic fashion.

For the Square Kilometre Array (SKA) (Dewdney et al. 2009), a key figure of merit is the so called survey speed (Bunton \& Hay 2004). This is a measure how fast SKA will be able to map out a given fraction of the sky with a given sensitivity. The survey speed is proportional to the product of the square of the instantaneous telescope sensitivity and the instantaneous Field-of-View (FoV) of the telescope. The cost per unit survey speed for a given system concept depends strongly on the operating frequency, making distinct sys- 
tem concepts the preferred option for distinct frequency bands. This paper deals with the design and building of a demonstrator called Electronic Multi Beam Radio Astronomy ConcEpt (EMBRACE). EMBRACE comprises an innovative step in providing low cost phased array technology for an aperture array option for SKA. It incorporates features like multiple independent beams, high aperture efficiency versus low side lobes, and a large FoV per beam. Most of these features are unique to aperture array technology. An overview of the station architecture is provided and details of the front end part of the system is discussed.

The digital processing part of a station is described in more detail by Picard (2010). Currently the two stations are being finalised. Wijnholds et al. (2010) present first results with the Westerbork EMBRACE station and first results at the Nançay EMBRACE site are discussed by Olofsson et al. (2010).

\section{System Architecture}

\subsection{Overview}

EMBRACE as a whole consists of two stations, one in Nançay in France and one in Westerbork in the Netherlands. Each station is a phased array antenna system which covers a frequency range from 500-1500 MHz. It provides two independent analogue beams of approximately 16 degrees beam width at $1 \mathrm{GHz}$. Both beams are capable to scan electronically more than 45 degrees from zenith using a combination of phase shifters and delay lines. The major array design requirements are summarised in Table 1.

A system level overview of one EMBRACE station is shown in Fig. 2. An EMBRACE station can be divided roughly into two parts: a front-end and a back-end. The front-end consists of the antenna array including the radome and the supporting mechanics for the array. The array is organised in tiles as primary building blocks. At the top in Fig. 2, the layout of a tile with the essential Radio Frequency (RF) beam former building block is shown. Each tile contains $2 \times 72$ antenna elements and has a size of $1.125 \mathrm{~m}^{2}$. The antenna design of EMBRACE incorporates two polarisations, however only the signals from one polarisation are electronically processed. In the picture it is shown that the antenna elements are oriented under 45 degrees with the edges of a tile and how the 144 elements are organised in a dual polarisation configuration. The antenna signals are beam formed into two, fully independent, RF beams. The resulting two beam signals are transported over coaxial cables to the back-end.

The back-end part is hosted in a small shielded shelter near the array. A block diagram of the back-end is shown at the right hand side in Fig. 2. The back-end contains the control subsystem and all the remaining electronics required for further processing of the RF signals coming from the tiles. First, the RF beam signals are down converted and digitised. In the digital domain, the signal is represented as a series of narrow band signals where after digital beams are formed. Finally, the system provides a WAN interface from which the resulting digital station beam signals can be transported over fibre to a remote correlator.
Table 1: EMBRACE Demonstrator Main Requirements

\begin{tabular}{|c|c|c|}
\hline Requirement & Remark & Value \\
\hline Number of stations & $\begin{array}{l}\text { In France and the } \\
\text { Netherlands }\end{array}$ & 2 \\
\hline $\begin{array}{l}\text { Total physical } \\
\text { collecting area } A_{\text {phy }}\end{array}$ & Both stations & $300 \mathrm{~m}^{2}$ \\
\hline Aperture efficiency & & $\geq 0.8$ \\
\hline Frequency range & & $500-1500 \mathrm{MHz}$ \\
\hline $\begin{array}{l}\text { System } \\
\text { temperature }\end{array}$ & @ $1 \mathrm{GHz}$ & $\leq 100 \mathrm{~K}$ \\
\hline $\begin{array}{l}\text { Instantaneous array } \\
\text { bandwidth }\end{array}$ & RF beams & $100 \mathrm{MHz}$ \\
\hline $\begin{array}{l}\text { Number of } \\
\text { analogue FoVs }\end{array}$ & RF beams & 2 \\
\hline Polarisation & Single linear & 1 \\
\hline $\begin{array}{l}\text { Half power beam } \\
\text { width }\end{array}$ & RFbeam @1 GHz & $>15 \mathrm{deg}$ \\
\hline Scan range $\theta$ & from zenith & $\geq 45 \mathrm{deg}$ \\
\hline Side lobe levels & $\begin{array}{l}\text { With respect to main } \\
\text { beam. No grating } \\
\text { lobes }\end{array}$ & $\leq-13.2 \mathrm{~dB}$ \\
\hline $\begin{array}{l}\text { Signal dynamic } \\
\text { range }\end{array}$ & $\begin{array}{l}\text { @ output A/D } \\
\text { converter }\end{array}$ & $\geq 60 \mathrm{~dB}$ \\
\hline $\begin{array}{l}\text { Digital output } \\
\text { bandwidth }\end{array}$ & per FoV & $\geq 40 \mathrm{MHz}$ \\
\hline $\begin{array}{l}\text { Number of digital } \\
\text { beams }\end{array}$ & per FoV & $\geq 8$ \\
\hline
\end{tabular}

In a balanced SKA design, front-end bandwidth (and thus FoV) needs to match with the back-end processing capacity of the central correlator, to obtain a cost effective implementation. Future upgrade possibilities should of course be taken into account. EMBRACE demonstrates how FoV can be tailored to bandwidth requirements by RF beam forming near the elements to reduce costs. The required FoV is achieved by organising the elements in units denoted as tiles. The first beam forming takes place on the elements of a tile. Therefore the FoV is defined by the size of a tile. Early in the system design stage, the system was divided in two main parts: the front-end and the back-end. The front-end is defined as the total set of tiles including the signal distribution between the tiles and the back-end.

The application of high scale integrated circuits is essential for cost reduction of systems in general. However, a large phased array system is inherently distributed over a large area. For any design, noise performance demands that (active) Low Noise Amplifiers (LNA's) have to be integrated in the array close to the elements. From this it is evident that the LNA's are no suitable candidates for high scale integration. All signals from the LNA's need to be distributed to a central location and on the other hand DC power needs to be distributed from 


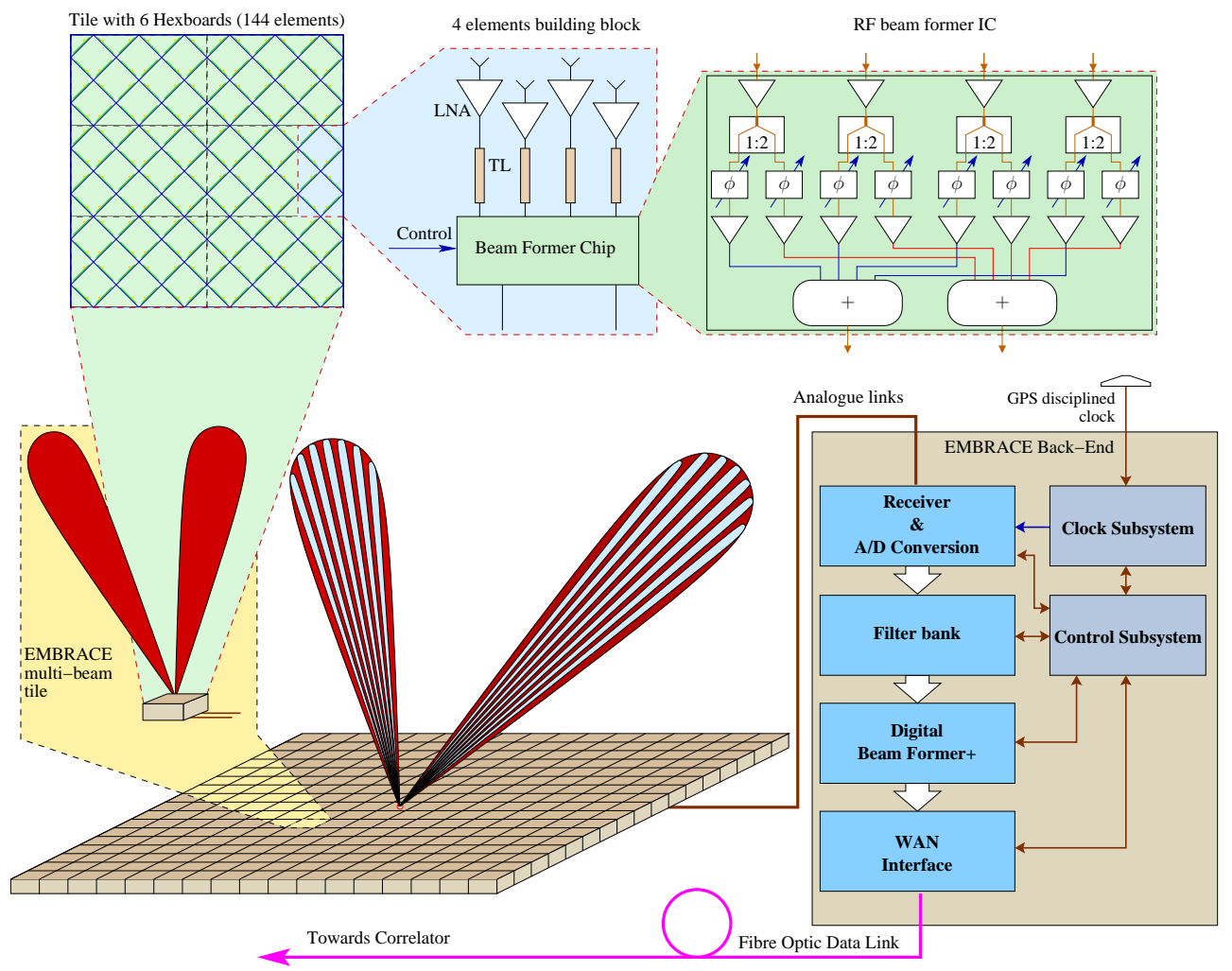

Fig. 2: System level overview of the EMBRACE station architecture. Antenna signals are combined at tile level using integrated circuit technology. Each tile provides two RF beam signals to a local processing facility where digital station beams are formed. Each tile uses two coaxial cables to transport the RF signals and also supply DC power and control signals to the tiles.

a central location to all LNA's. The signal distribution tree is narrowed already near the elements directly after the LNA's. The first signal combination is performed by a large scale integrated beam former chip, which combines the signals from four elements and provides two RF beam outputs. The signal distribution tree is narrowed further on a tile by combinations of all the remaining signals into two RF tile beam signals. In EMBRACE the number of required cables is reduced by multiplexing DC power, control signals and the analogue RF beam signals towards the back-end.

Whereas the front-end forms a large distributed sub-system by nature, the back-end can be a centralised system. The backend handles frequency conversion, analogue to digital conversion and digital station beam forming. This is an important design choice. Centralising the analogue to digital conversion avoids the distribution of additional clock and Local Oscillator (LO) signals towards the tiles. The LO system is described in more detail by Bianchi (2010). Signal transport between front-end and back-end is implemented with one coaxial link per RF beam per tile. These RF analogue links between the tiles and the back-end processing simplifies the tile design and totally decouples the antenna from the receiver. This reduces EMC related problems in general, since the LO and clock signals now have to be distributed in the back-end cabinet only. Furthermore, in the back-end all sensitive analogue electronics can be shielded from the digital electronics. A system with analogue to digital conversion (and frequency conversion if re quired) in the front-end would not allow for such a clear separation.

The antennas are designed in a dual polarised fashion, however only a single polarisation is used. This configuration includes all electromagnetic effects of a dual polarised antenna. From a technical point of view the step to build a front-end, capable to process two polarisations, is just a matter of doubling the front-end electronics with respect to one polarisation.

\subsection{Tile Architecture}

The array is organised in tiles as primary building blocks. Each tile consists of six identical hex boards and one centre board, as shown in Fig. 3. A hex board has 24 antenna elements, of which 12 are amplified and phase shifted using beam former chips. Each beam former chip combines the signals from four elements and creates two independent outputs. The centre board sums the outputs of the hex boards and distributes DC power and control to the hex boards. On each tile, the antenna signals are beam formed into two, fully independent beams at RF level. The resulting two beam signals are transported over coaxial cables to the back-end. 


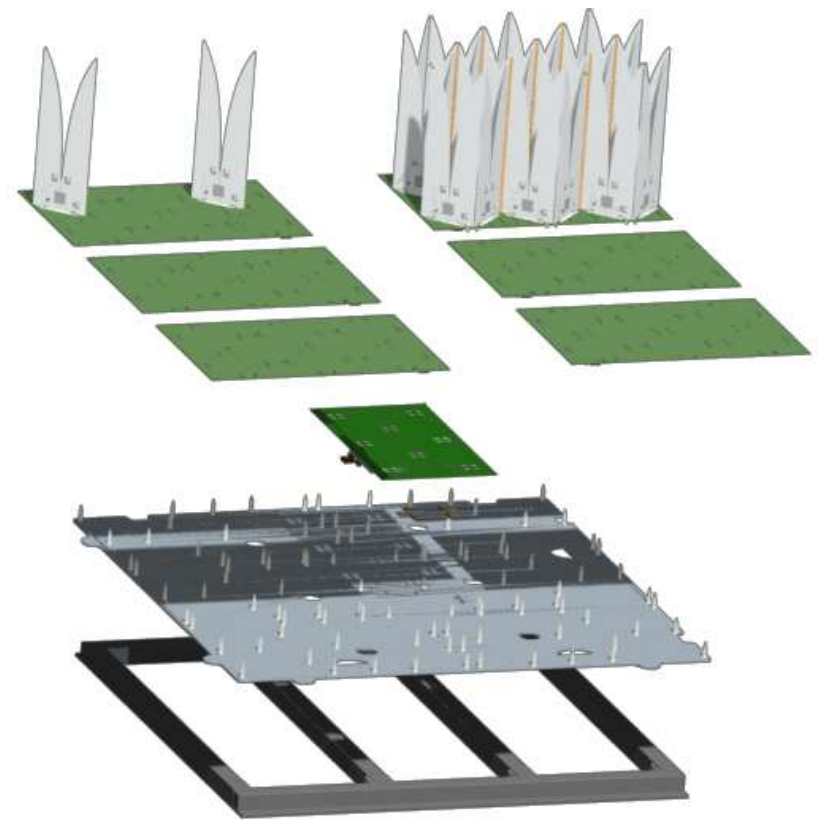

Fig. 3: Exploded view of a single EMBRACE tile, showing antenna elements, hex boards and centre board as well as mechanical support frames

\subsection{Scanning Beams}

In an aperture array system the resulting station beams are steered by compensating the differences in time of arrival for a wave front at each antenna. This compensation can be implemented using delay lines or phase shifters. Delay lines and phase shifters each have its benefits and limitations. Within EMBRACE the main design drivers are bandwidth and the possibility of integration. Delay-lines are perfect for bandwidth, however they are hard to integrate in an RF Monolithic Microwave Integrated Circuit (MMIC). Integrated solutions are either limited by noise (RC networks) or physical dimensions (LC networks or pure true time delays). Phase shifters can relative more easily be integrated, but they have bandwidth limitations. The problem arises due to the fact, that phase shift can only approximate time delay over a small frequency range $(\tau=-\varphi / \omega)$. Over larger frequency ranges, the scanned beam will start to squint.

Embrace takes a hybrid approach, to combine the best of both worlds. The first combination step uses a phase shifter. In this stage we still have a broad beam width, so squint does not cause significant gain loss. After combining 12 antennas, time delays are introduced. These are implemented as one-bit delays, either the delay or a bypass path is used. These are placed on the hex boards and the centre board. After the combination of 72 antennas from one tile, the signal is digitised and further beam forming is performed in the digital domain. First, the beam signal is decomposed as a series of narrow band signals by a poly phase filter bank to allow for beam forming by phase shifters in the digital domain. Optionally it is possible to combine the resulting beam signals from four tiles to reduce the number of required digital processing signal inputs.

\subsection{System Dynamic Range}

The dynamic range of the system is determined by two bounds. At the low end, it is limited by noise and at the high end the system is limited by non-linearity of the (active) components.

The system noise figure is determined by several factors. It is a summation of noise contributions starting at the antenna enclosure, the resistive losses of the antenna element, the total front-end up to the A/D converter. Its main contribution is primarily dominated by the first stage in the front-end, the LNA. However, a compromise is required between linearity and system noise figure. More gain in the systems improves the noise figure, but it degrades linearity of the system. The EMBRACE locations at both Westerbork and Nançay cannot be regarded as Radio Frequency Interference (RFI) quiet regions. Broadband systems such as EMBRACE have to cope with the RFI in the received frequency band. This results in strong linearity requirements. Increasing linearity of the active devices results in considerable additional power consumption and dissipation is sues.

Looking at the noise contributions of the EMBRACE system, it is interesting to note that from the total noise figure $77 \%$ is coming from the part up to the output of the first LNA. The second stage still adds $7 \%$ and still $16 \%$ is contributed by the rest of the RF chain. This results from the gain distribution dominated by RFI requirements and a power budget of about $100 \mathrm{~W} / \mathrm{m}^{2}$.

\subsection{LNA Noise}

It is well understood that the noise figure of an LNA is dependent on the source impedance of the antenna Gonzales (1984). The noise behaviour can be described by

$$
F=F_{\min }+4 r_{n} \frac{\left|\Gamma_{\mathrm{src}}-\Gamma_{\mathrm{opt}}\right|^{2}}{\left(1-\left|\Gamma_{\mathrm{src}}\right|^{2}\right)\left|1+\Gamma_{\mathrm{opt}}\right|^{2}},
$$

where $F_{\min }$ is the minimal noise figure, $r_{n}$ is the normalised noise resistance, $\Gamma_{\mathrm{src}}$ is the source impedance and $\Gamma_{\mathrm{opt}}$ is the optimum noise impedance.

For a system with multiple inputs, like an antenna array system, the resulting source impedance which drives each LNA is not obvious. It can be shown that the active impedance needs to be considered as the driving source impedance for each LNA (Ivashina et al. 2008). The active reflection coefficient of antenna port $i$ can be written as

$$
\Gamma_{\mathrm{act}, i}=\left.\frac{1}{w_{i}} \sum_{j=1}^{N} w_{j} S_{\mathrm{ant}, j, i}\right|_{Z_{0}=Z_{\mathrm{LNA}}},
$$

where $w_{i}$ is the complex weight of each channel and $S_{\text {ant }}$ is the scattering matrix describing the antenna array. Since each beam direction has a unique set of weights, the active reflection coefficient is dependent on beam steering and thus the noise figure of the system will be influenced by beam steering. So for a low noise phased array system, not only $\mathrm{NF}_{\text {min }}$ determines the resulting noise performance. Besides $\mathrm{NF}_{\min }$, both the active reflection coefficient (determined by the array) and $r_{n}$ (a property 


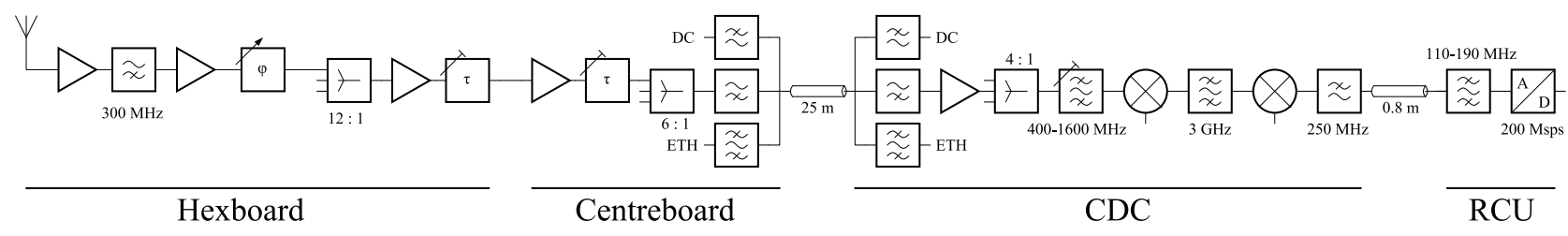

Fig. 4: The system block-diagram. Only a single chain is shown. Point where multiple signals are combined are indicated.

of the LNA), need to be taken into account for system noise performance analysis.

\section{System Implementation}

Following the signal path shown in the block diagram of Fig. 4 the implementation is discussed. The discussion starts however with the radome which is not shown in the figure.

\subsection{Radome}

To protect the electronics from the outside environment, such as sun, wind, rain, snow, a radome has been designed and build for each station. The total size of a radome is $(\mathrm{L} \times \mathrm{W} \times \mathrm{H})$ $20 \mathrm{~m} \times 20 \mathrm{~m} \times 4.2 \mathrm{~m}$ for each station. The radome should be capable of handling extreme weather conditions in the Netherlands, $0.5 \mathrm{~m}$ of snow and wind condition beyond wind force 12 . Furthermore, it also provides a thermal barrier to prevent thermal shocks.

The mechanical requirements in general are not compliant with the desired behaviour for radio frequencies. Here a radio 'transparent' enclosure is required. Also no obstructions are allowed within the scan range. A good low cost material choice in combination with clever structural solutions, combine the aforementioned requirements. Electrical properties of the materials have been verified by using an high $\mathrm{Q}$ waveguide cavity (TE110-mode), filled with the material. Both dielectric constant and dissipation factor showed excellent RF behaviour.

To test the concept, a small demonstrator of $4 \mathrm{~m} \times 4 \mathrm{~m}$ was build. Both electrical and mechanical test proved to be successful. Material choice and construction are currently part of a patent application.

A measurement campaign has been performed at the Westerbork site, to asses the RFI situation. This showed that the most dominant RFI sources originates from a single location: A 300m high broadcast tower, at approximately $10 \mathrm{~km}$ distance. The fact that most RFI comes from a single location, can be used to our benefit. Since the antenna pattern has a null at the horizon in the H-plane, we can direct the antenna, so that its null is pointing towards the television tower, the RFI can be suppressed. Thereby abandoning the traditional east-west alignment of the WSRT. Since the radome orientation is coupled to the antenna rotation, its orientation is also set.
Table 2: Design parameters for the bilateral Vivaldi array with strip line feed.

\begin{tabular}{|l|l|}
\hline Parameter & Target value \\
\hline Frequency range & $400-1400 \mathrm{MHz}$ \\
\hline Array bandwidth & $\geq 3: 1$ \\
\hline Element separation & $12.5 \mathrm{~cm}$ \\
\hline Scan range $\theta$ & $\geq 45 \mathrm{deg}$ \\
\hline Dielectric thickness & $1.6 \pm 0.2 \mathrm{~mm}$ \\
\hline Relative dielectric constant & 4.3 \\
\hline Loss tangent & 0.02 \\
\hline Reference impedance & $50-100 \Omega$ \\
\hline VSWR (active reflection) & $\leq 2$ \\
\hline
\end{tabular}

\subsection{Antenna array}

\subsubsection{First Array Prototype}

Since 1995, an R\&D programme has been conducted at ASTRON regarding wide band aperture array technology for SKA. A range of demonstrators have been developed and the precursor of EMBRACE was the successful design of the Thousand Element Array (THEA) (Smolders \& Kant 2000; Schaubert et al. 2000; Hampson \& bij de Vaate 2001; Boryssenko \& Schaubert 2001). With THEA, a wide band phased array based on Vivaldi radiators was successfully demonstrated. Therefore, the Vivaldi radiator was again chosen as the appropriate radiator to produce a scanning array over a bandwidth of greater than 3:1 ratio.

As the demonstrator stations will be large phased arrays $(\geq 20 \lambda \times 20 \lambda)$, it is appropriate to design it using an infinite array approach. A Periodic Boundary Finite Difference Time Domain (PB-FDTD) code developed by Holter \& Steyskal (2002), has been used in the design of the array. It is known to give accurate results on Vivaldi arrays and has been has been extensively used elsewhere and is known to produce excellent results (Holter et al. 2000).

The design of a phased array starts with the scanning requirement at the highest frequency of operation. This provides the elemental separation, producing a radiation pattern free of grating lobes, within the visible range. Analogue RF beam forming is performed on the signals from the elements within one tile. The FoV of the resulting RF beams is inversely proportional with the size of the tile. A beam width of about 16 degrees at $f=1 \mathrm{GHz}$ on tile level was set as a requirement demonstrating a relative large FoV. It follows that with a ele- 


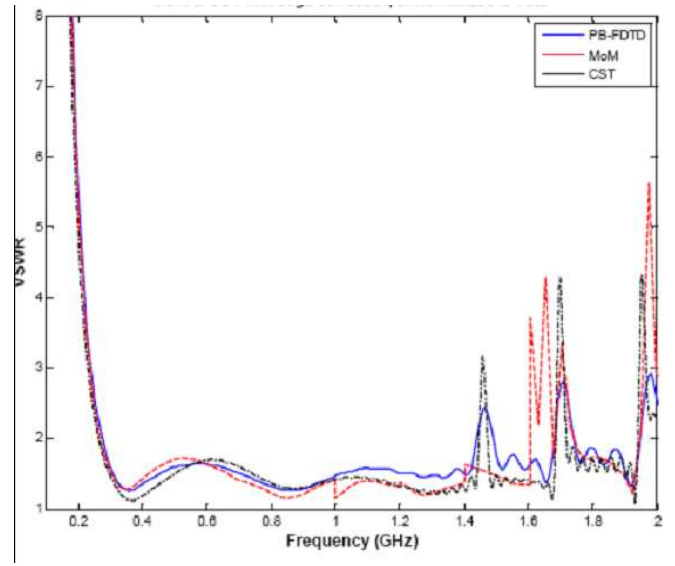

Fig. 5: Reflection coefficient versus frequency at broadside of the first prototype array

ment separation of $12.5 \mathrm{~cm}$, an $8 \times 8$ element array can meet the scanning requirement up to 45 degrees at $f=1.4 \mathrm{GHz}$ in combination with the FoV requirement. The design parameters for the bilateral Vivaldi array with strip line feed are summarised in Table 2. The Vivaldi array was optimised using the PB-FDTD code and the results were verified using several other codes.

In Fig. 5, VSWR plots of the final result are shown as function of frequency obtained with PB-FDTD, Method of Moments code from Chio \& Schaubert (2000) and commercially available EM software from CST. From Fig. 5 it is clear that there is a high degree of correlation in the results.

\subsubsection{Aluminium Vivaldi Radiator Design with Microstrip Feed Designed on Rogers Material}

The first array prototype design has two major drawbacks. Losses in the dielectric of FR4 are too high to be able to meet the noise requirement. Secondly, the first array prototype is a single polarisation design. Going to more expansive microwave material for the elements would render an implementation out of cost bounds, so this is no option. To overcome both issues along the path of cost reduction, the design of the tile is changed by replacing the FR4 elements with a radiator made of solid but a fairly thin sheet of aluminium with a microstrip feed. Furthermore the step was made from a single polarisation configuration to a dual polarised element configuration. Analysis with the PB-FDTD code showed that both the linearly and dual polarised antenna are easily achievable with very similar refection coefficient versus frequency curve. This time the cost and the long term effects of the material have also been taken into consideration. Although EMBRACE requires a linearly polarised array only, the electromagnetic design is dual polarised. The aluminium radiator is fed with a microstrip feed designed using Rogers 4003 material $\left(\varepsilon_{r}=3.38\right)$ which, although very small, is the most costly item on the antenna and hence there may be further opportunities for cost reduction in the future. As the microstrip line on the feed board is in front of the LNA, such low loss substrate is essential to meet the noise requirements.

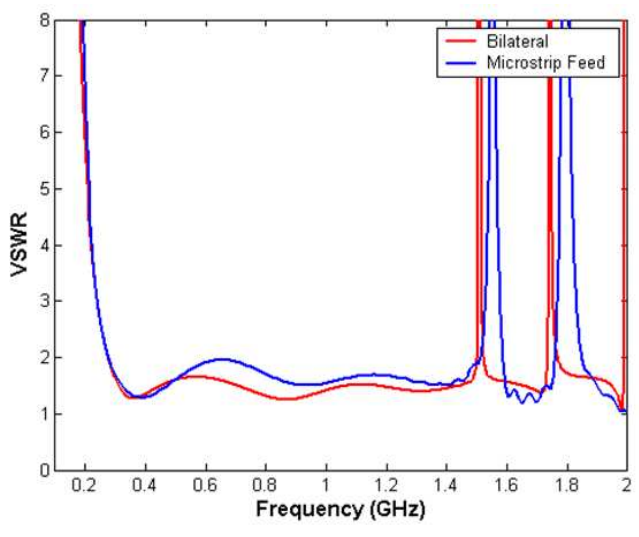

Fig. 6: VSWR versus frequency for a aluminium Vivaldi array fed by a microstrip feed compared with the original bilateral Vivaldi design.

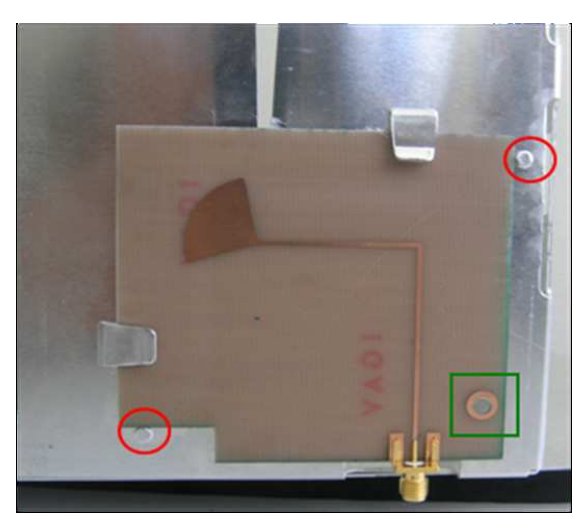

Fig. 7: Close up of the feed arrangement on the radiator. In the circles are the pawls for alignment. In the square the rivet is placed to fixate the board.

Using a geometry similar to the the original bilateral Vivaldi design, the simulation results of the final reflection coefficient aluminium radiator fed with a microstrip feed is shown in Fig. 6.

A detailed arrangement of the feed on the radiator is shown in Fig. 7, where the alignment pawls and clips to hold the feed in place are clearly visible. The feed board is finally fixated on the radiator by means of a rivet. With the arrangement of Vivaldi radiators made out of thin but solid aluminium sheets and microstrip feeds on a Rogers 4003 material, the design was considered to be fairly close to optimum in sense of cost and performance for the demonstrator. An extensive analysis on the behaviour of the array for broadside several scan directions was therefore carried out on this particular configuration of the feed and radiator. This included the effects of the assembly holes in the radiators. The results shown in Fig. 8, resemble very close the results obtained from the linearly polarised case. A prototype tile based on this dual polarised aluminium Vivaldi array has been build and evaluated. 


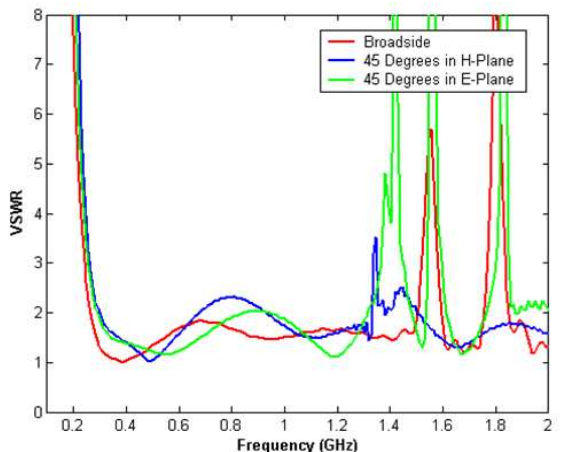

Fig. 8: VSWR vs. frequency at broadside and $45 \mathrm{deg}$ in $\mathrm{H}$ and $\mathrm{E}$ Planes of a dual polarised array modelled with assembly holes.

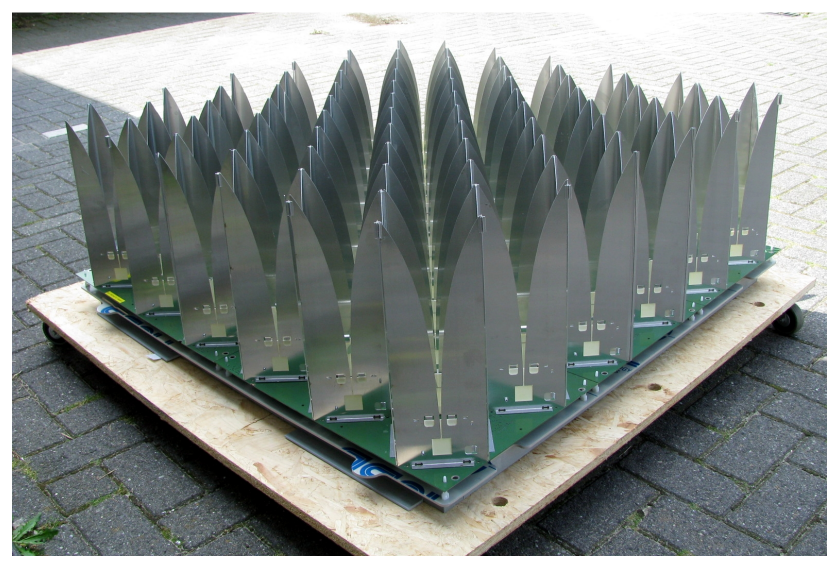

Fig. 9: An EMBRACE tile of the final design with aluminium Vivaldi radiators in a dual polarisation configuration.

\subsubsection{Final Array Design}

The array is designed using infinite array assumptions. As previously mentioned in section 2 , the demonstrator array is organised in tiles. Therefore, the array hardware should maintain electrical continuity between the tiles. In order to achieve this continuity in practise, the radiating elements on the final tile design were oriented at 45 degrees angles with the tile edges. The final tile design contains $2 \times 72$ antenna elements and its final size is $72 / 64 \mathrm{~m}^{2}$. A picture of the final tile is shown in 9 . The mechanical design of the radiator has also evolved a step further. In the final design single radiator elements are connected together using extruded profiles between the elements.

\subsection{Low Noise Amplifier}

As explained in section 2.6, both the active reflection coefficient and $R_{n}$ should deserve equal, if not more attention. The $R_{n}$ is dominated by the transistor itself. A market survey of transistors, that combine a low $R_{n}$ in combination with a low $\mathrm{NF}_{\text {min }}$ and reasonable matching, resulted in one of Avago's E-pHEMT devices. Any components between the LNA and the antenna terminal will either limit bandwidth or increase noise. The solution was found by choosing a proper reference impedance at the antenna terminal. $70 \Omega$ proved to be a good compromise between noise behaviour, reflection and manufacturability. With the $70 \Omega$ reference impedance only a small series inductance is needed in series with the input for stability reasons. In combination with resistive feedback stability is ensured.

\subsection{Beam Former Chip}

An analogue RF MMIC has been developed, to control the phases of each element in an EMBRACE tile. To generate phase shift, the design uses a vector modulator approach, with a filter network network generating the four main phase states $0,90,180$ and 270 degrees. Smaller phase steps can be created by combining these vectors.

The chip has been designed by OPAR, France in a QUBIC4G BiCMOS $0.25 \mu \mathrm{m} \mathrm{SiGe} \mathrm{process} \mathrm{of} \mathrm{NXP.} \mathrm{With} \mathrm{this}$ chip two times four differential channels are independently phase shifted, summed and amplified. Amplitude control is used to compensate for gain variation of the chip. The phase settings are set by a digital serial control interface, to reduce layout complexity and crosstalk issues.

\subsection{Control and Down Conversion Unit}

As mentioned before the CDC plays a central role. It converts the RF band to IF with a super-heterodyne mixing scheme. A $3 \mathrm{GHz}$ intermediate frequency has been chosen to create enough spectral distance for image suppression using a $100 \mathrm{MHz}$ wide band pass filter.

\subsection{Receiver Unit}

EMBRACE reuses the receiver unit of LOFAR. Following the RF path at the input, the signal is first preconditioned to the correct signal to noise ratio for conversion with the A/D converter. After pre-conditioning a very high quality bandpass filter selects the correct band, removing out-of-band spurious.

Finally, a 12-bit $200 \mathrm{Msps}$ A/D converter digitises the signal. The system uses under-sampling in the second Nyquist zone. The effective input frequency range is 110 to $190 \mathrm{MHz}$.

\subsection{Array control and supply architecture}

Each tile can be controlled independently using raw Ethernet packages. The point-to-point communication uses one of the two coaxes that connect the tile to the back-end. Furthermore the tile only listens (slave). The communication uses a $10 \mathrm{Mbit} / \mathrm{s}$ link thus filling the lower part below the received spectrum of the coax. Separation between the communication and the measured frequency bands is ensured by an active circulator and filters. This part was designed and tested by our partners of the Max Planck Institute.

A phantom supply architecture is selected to distribute power to the tiles. Due to limited current capability of the cables and connectors a high voltage of $48 \mathrm{~V}$ has been chosen. Additional filtering on the CDC back plane will reduce spurious signals such as DC/DC noise considerably. 


\section{Conclusions}

EMBRACE has been developed in the context of the European FP6 project SKADS as a collaboration between several European partners. In this paper we presented the design and the realisation of EMBRACE, a phased array technology demonstrator for SKA. The main design choices are discussed and an overview of the implementation of the EMBRACE stations is given. More details on parts of the system including first results obtained with the EMBRACE stations are addressed in several other papers of the SKADS conference 2009.

\section{References}

Bianchi, G. 2010, in Widefield Science and Technologies for the SKA, ed. S. A. Torchinsky et al.

Boryssenko, A. O. \& Schaubert, D. H. 2001, in Antennas and Propagation Society International Symposium, IEEE, Vol. 2, 436-439

Bunton, J. D. \& Hay, S. G. 2004, Experimental Astronomy, 17, 381

Chio, T.-H. \& Schaubert, D. H. 2000, IEEE Transactions on Antennas and Propagation, 48, 879

Dewdney, P. E., Hall, P. J., Schilizzi, R. T., \& Lazio, T. J. L. W. 2009, Proceedings of the IEEE, 97, 1482

Gonzales, G. 1984, Microwave Transistor Amplifiers: Analysis and Design (Englewood Cliffs (New Jersey), USA: PrenticeHall Inc.)

Hampson, G. A. \& bij de Vaate, J. G. 2001, in European Microwave Conference, 1-4

Holter, H., Chio, T.-H., \& Schaubert, D. H. 2000, IEEE Transactions on Antennas and Propagation, 48, 1707

Holter, H. \& Steyskal, H. 2002, IEEE Transactions on Antennas and Propagation, 50, 1725

Ivashina, M. V., Maaskant, R., \& Woestenburg, B. 2008, Antennas and Wireless Propagation Letters, IEEE, 7, 733

Olofsson, A. O. H. et al. 2010, in Widefield Science and Technologies for the SKA, ed. S. A. Torchinsky et al.

Picard, P. 2010, in Widefield Science and Technologies for the SKA, ed. S. A. Torchinsky et al.

Schaubert, D. H., Chio, T.-H., \& Holter, H. 2000, in Antennas and Propagation Society International Symposium, IEEE, Vol. 1, 178-181

Smolders, A. B. \& Kant, G. W. 2000, in Antennas and Propagation Society International Symposium, 2000. IEEE, Vol. 1, 162-165

Wijnholds, S. J., Kant, G. W., van der Wal, E., et al. 2010, in Widefield Science and Technologies for the SKA, ed. S. A. Torchinsky et al. 